\title{
Empowering Qualitative Research Methods in Education with Artificial Intelligence
}

Luca Longo

Technological University Dublin, luca.longo@tudublin.ie

Follow this and additional works at: https://arrow.tudublin.ie/scschcomcon

Part of the Computer Sciences Commons

\section{Recommended Citation}

Longo L. (2020) Empowering Qualitative Research Methods in Education with Artificial Intelligence. In: Costa A., Reis L., Moreira A. (eds) Computer Supported Qualitative Research. WCQR 2019. Advances in Intelligent Systems and Computing, vol 1068. Springer, Cham. DOI: 10.1007/978-3-030-31787-4_1

This Conference Paper is brought to you for free and open access by the School of Computer Sciences at ARROW@TU Dublin. It has been accepted for inclusion in Conference papers by an authorized administrator of ARROW@TU Dublin. For more information, please contact arrow.admin@tudublin.ie, aisling.coyne@tudublin.ie,gerard.connolly@tudublin.ie.

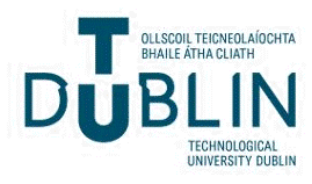


See discussions, stats, and author profiles for this publication at: https://www.researchgate.net/publication/335868951

\section{Empowering Qualitative Research Methods in Education with Artificial Intelligence}

Conference Paper · January 2020

DOI: 10.1007/978-3-030-31787-4_1

CITATIONS

READS

5

3,259

1 author:

Technological University Dublin - City Campus

88 PUBLICATIONS 1,024 CITATIONS

SEE PROFILE 


\title{
Empowering qualitative research methods in education with artificial intelligence
}

\author{
Luca Longo ${ }^{\star \star}$ \\ School of Computer Science, \\ Technological University Dublin, \\ Republic of Ireland \\ Iuca.longo@tudublin.ie
}

\begin{abstract}
Artificial Intelligence is one of the fastest growing disciplines, disrupting many sectors. Originally mainly for computer scientists and engineers, it has been expanding its horizons and empowering many other disciplines contributing to the development of many novel applications in many sectors. These include medicine and health care, business and finance, psychology and neuroscience, physics and biology to mention a few. However, one of the disciplines in which artificial intelligence has not been fully explored and exploited yet is education. In this discipline, many research methods are employed by scholars, lecturers and practitioners to investigate the impact of different instructional approaches on learning and to understand the ways skills and knowledge are acquired by learners. One of these is qualitative research, a scientific method grounded in observations that manipulates and analyses non-numerical data. It focuses on seeking answers to why and how a particular observed phenomenon occurs rather than on its occurrences. This study aims to explore and discuss the impact of artificial intelligence on qualitative research methods. In particular, it focuses on how artificial intelligence have empowered qualitative research methods so far, and how it can be used in education for enhancing teaching and learning.
\end{abstract}

Keywords: Artificial Intelligence, qualitative research, methods, data analysis, education, teaching, learning, behaviourism, constructivism, cognitivism, automated reasoning, knowledge representation, machine learning, planning, perception, natural language processing

\section{Introduction}

Artificial Intelligence has been disrupting many sectors and disciplines, offering tools to conduct research and support innovation that were not available a couple of decades ago. This has been facilitated by the technological progress and the availability of instruments and technolologies for collecting, storing and analysing data of many forms. Artificial Intelligence has contributed to many fields and disciplines, accelerating scientific discovery [32. It has been informing natural language research [50], the social sciences [83, medicine [106 38,

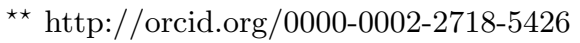


finance [3], psychology and human behaviour research [105], neuroscience [40] just to mention a few. However, there is a discipline in which the potential of artificial intelligence has not been fully explored and explored yet: education. The key goal of education is to design approaches, methodologies, methods and techniques for enabling learning and facilitating the acquisition of knowledge and skills. Many educational methods are used within education with teaching the core one. Teaching can occur in many forms and it is the aim of pedagogy to investigate different methods and how they can affect and enhance learning [18. In contrast to other disciplines where quantitative research methods are frequently employed, education and pedagogy heavily rely on qualitative methods to extract patterns from non-numerical data and allow inferences.

As a researcher in artificial intelligence and computer science as well as a third-level pedagogist, I strongly support the use of technology in general, and AI-based tools and techniques in particular to support teaching and promote learning. This article is intended for the broad audience of pedagogists, psychologists, social scientists and educational practitioners, on average not having the formal and technical skills usually belonging to engineers and computer scientists. Its aim is to informally define artificial intelligence and its main goals, so as to provide readers with the basic and core components of this fast growing discipline. Subsequently, the learning theories mainly used within education by pedagogists are introduced and the main qualitative research methods generally used by them are briefly described. A discussion follows, showing how the theoretical and practical advances in artificial intelligence have empowered qualitative research data analysis methods so far. Eventually, it proposes ways of how these advances can advance pedagogy in particular, through specific contributions to each educational learning theories (figure 21.

\section{Background}

Artificial intelligence, education and qualitative research methods have long histories and for those scholars working mainly in one of them, some notions and concepts belonging to the others might not sound very familiar. For this purpose, the content of this section is aimed at providing readers with the relevant background information and at describing the main core notions and concepts behind each of these fields of research.

\subsection{Artificial intelligence}

Artificial Intelligence (AI) can be traced back to 1943 when McCulloch and Pitts proposed a model of artificial neurons inspired by the physiological and functional properties of a neuron in the brain, and building on the propositional logic of Russell and Whitehead as well as the theory of computation brought forward by Alan Turing [80. From this, artificial intelligence has tremendously evolved and significantly extended its boundaries. It has seen early achievements and 


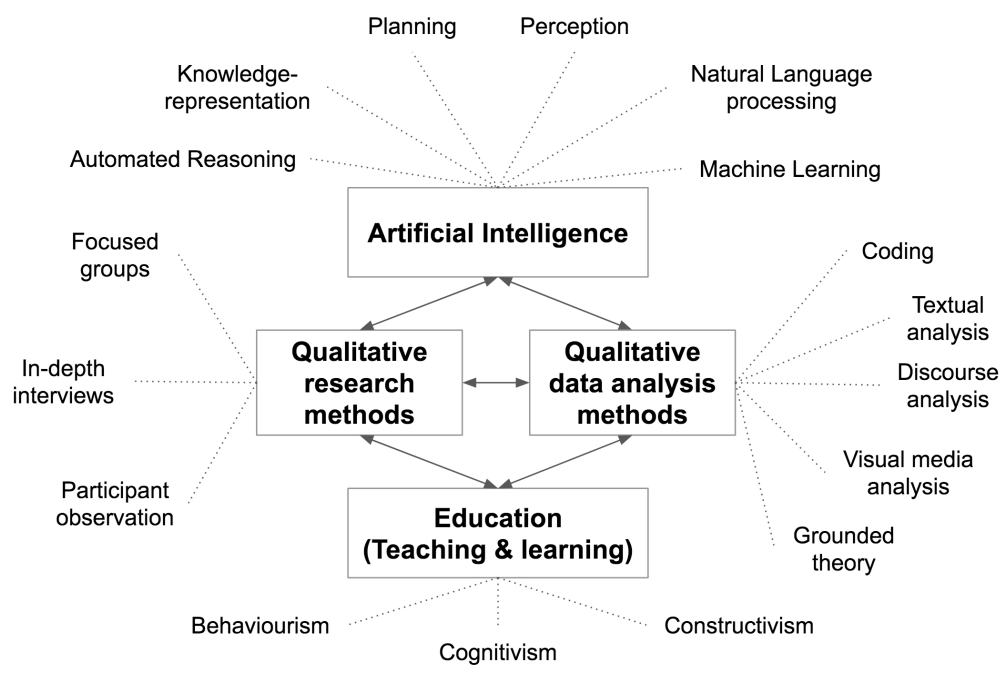

Fig. 1: Exploration of the impact of artificial intelligence for empowering qualitative research, its data analysis methods in education, teaching and learning

great expectations but it has also gone through difficult times [9. Early research was mainly devoted to knowledge-based systems and the development of expert systems enabling the commercialisation of tools for supporting various tasks in industrial settings. After a period known as the AI winter, in middle 80s, neural networks returned to the scene, and with the re-design of the back-propagation algorithm, they posed the basis of modern machine learning [58. In recent years, AI has gone through a revolution concerning the methodologies employed in research and the actual content being used for research. It is now more common to exploit existing theories for novel applications rather than proposing new ones. Late 90s have seen the emergence of intelligence agents to solve the main sub-problems of AI and then, in the early 2000s, with the availability of large datasets and computational power, it become norm to tackle these problems with a stronger emphasis on data and less on the theoretical approach to apply. Defining AI is not a trivial task since many definitions have been proposed by many scholars in the last 70 years. However, a set of major goals have emerged within AI: knowledge representation, automated reasoning, automated planning, machine perception, machine learning, natural language processing.

Knowledge-representation focuses on the formal representation of information about the world in a form that can be actually used by computers for solving tasks. It is a branch of AI that is connected to psychology because it builds upon the capabilities of humans to represent knowledge and information as well as to solve problems employing it [104]. Examples includes ontologies [76], frames and rules [124, semantics nets [25]. Automated reasoning is another branch of AI concerning logic and reasoning, strictly connected to the goals of knowledge 
representation. It is aimed at designing and building intelligent agents with automated reasoning capabilities. Example includes reasoning under uncertainty [98, defeasible and non-monotonic reasoning [70] as well as argumentation [67. Automated planning is a sub-field of AI devoted to the design and development of automated scheduling strategies or sequences of actions [31. This usually concerns the development of intelligent agents that aim to find optimal solutions in a complex multi-dimensional space and it is often connected to decision theories. Applications include planning and scheduling for autonomous vehicles or mean of transportations 90, reconfigurable production systems [10311] and in general distributed systems [121120. Machine perception is that sub-field of AI that concerns the construction of artificial systems able to interpret data that resembles the human way through the use of senses and the context around them 134. With the technological progress and the explosion of sensor-based technologies, early systems that were focusing on analysing data, mainly collected through keyboards and mouse [72], have now been empowered with a wider range of sensory input that is close to the way humans perceive, including computer vision [28], machine hearing [75], and machine touch [13. Machine learning is probably the fastest-growing sub-field of AI concerned with the development of statistical models and algorithms that are able to perform specific tasks without the use of explicit rules, instructions and directives [6]. Machine learning have seen a tremendous acceleration of research outputs and have significantly informed and contributed to the advance of the other sub-branches of artificial intelligence [47. Some of the plethora of applications includes classification of facial expressions [4] or cancers [22], forecasting in stock markets [4] and image processing [123] just to mention a few. Eventually, natural language processing is aimed at designing and developing artificial agents that are capable of interpreting and processing natural language. Example of problems includes speech recognition [42], natural language generation [108] and machine translation [51].

\subsection{Qualitative research methods}

A number of research methodologies and methods are employed by scholars in science. One of these is qualitative research methods [135. These are scientific methods of observation that, in contrast to quantitative research methods, make use of non-numerical data such as textual information, images, audio and video. It is not grounded on the occurrences of things or their frequencies or measures. Rather it focuses on investigating why and how a phenomenon takes place, by understanding the meaning of concepts, definitions and features of things. The core element is the descriptive observations made by scholars and its main strength lies in its ability to provide complex textual descriptions of how people experience things. Qualitative research has a very long history and its literature is very

fast that would be impossible to review all of its methods. However, a number of classes of qualitative research methods have been identified. These are referred to as semi-structured methods and include participant observations, in-depth interviews and focus groups [99]. 
Participant observation is a method for collecting data on naturally occurring behaviours within their usual contexts [126. The goal of this method is to get a richer familiarity with a group of people while interacting in their cultural environments, usually over an extended period of time [89. For example, within education, observation can be conducted through analysis of documents produced by learners in the classroom or other environemts 7. In-depth interviews are more relevant on an individual basis, when the researcher is interested in the history of a learner, her/his own perspectives and experiences 60. Considerable effort is spent by the researcher to grasp the essence of the phenomenon under investigation and to use literary devices to transmit it as rich as possible [143. Focus group is a method that focuses on eliciting data on the cultural norms of a group of people. The main goal is to shape broad overviews and summary of certain issues concerning to a group or represented subgroups [137. The purpose is to promote a comfortable atmosphere of disclosure of information by people who can share their beliefs, ideas, attitudes and experiences about a topic [142].

The format of the questions usually administered by a qualitative researcher is open-ended. Thus the main type and shape of the data produced by the application of qualitative research methods are field notes, audio, sometimes video, recordings, transcripts and images. These methods seek to explore hypotheses about a phenomenon rather then confirming them. Data collection instruments are flexible as well as those approaches and techniques used for eliciting and categorising responses to questions. The main goal is to describe variation, explain relationships, investigate individual experiences and group norms. Some aspects of research studies are adjustable, as the addition or exclusions of questions or their rewording. The responses by participants might impact the subsequent questions. In nature, the design of a qualitative research study is iterative: it evolves and can be updated according to data and what is learned 63.

\subsection{Qualitative data analysis methods}

A frequently used method to analyse qualitative data is qualitative coding. It refers to that process of assigning descriptive labels to pieces of data with the aim of helping researchers in the development of their theories and hypotheses 2. It is a process of organising qualitative information in a systematic order employing techniques such as linkage, grouping, aggregation to support meaning extraction and formulation. Unfortunately, this process is often time-consuming as it demands scholars to analyse data with great precision in order to identify interesting patterns, assign descriptive labels and categories according to commonly shared characteristics [116. However, with the technological progress and the plethora of technologies that can be employed for data gathering, the availability of data is exponentially growing. Therefore, for researchers it would be impossible to go through thousands of records containing qualitative information. This would not support the identification of inconsistencies within data and the development of robust hypotheses. 
Coding enables content analysis, a strict and systematic process of summarising and reporting written data composed by a set of procedures for the rigorous investigation, analysis and verification of the contents [79] for making replicable and valid inferences [54] of any written communicative materials, such as documents, interviews transcriptions, speeches, intended for people other than the research analyst. It often uses categorisation as a means to reduce large quantities of data. In a nutshell, content analysis involves coding, categorising the unit of analysis such as words and sentences in to meaningful categories and descriptive labels, comparing and linking them as well as concluding by drawing theoretical conclusions and hypotheses. In detail, content analysis starts with the definition of a research question and the population from which the unit of analysis of text are to be sampled. A strategy for defining the sample of interest is set according to the notions of representativeness, access and generalisability. It follows a definition of the context of the generation of the document which includes, for instance, the analysis of who was involved in data collection/generation/transcription, origins of documents, corroboration and authenticity of data. Then the unit of analysis has to be decided as well as the codes to be used in it enabling the construction of the categories for grouping key features of the written data. Subsequently, coding and categorisation of data has to be performed as well as its analysis that includes activities such as extrapolation of trends, patterns and differences, standards and indices as well as linguistic representations. These often produce numerical tabular data that can be used for statistical analysis by employing quantitative research methods. Eventually, summarisation follows with the researcher making speculative inferences and generating theories and hypotheses [18.

Beside content analysis other approaches exist that do not fragment the text as in coding. The assumption here is that words can carry many meanings thus their nuances are context dependent and their separation is not always the best approach. Example of contexts include conversations, narratives and autobiographies, specific types of discourses. In discourse analysis, words and sentences are semantically linked to each other and their meaning is influenced by those before and after 30. Qualitative research analysis can be conducted also on visual media such as images and videos. They are a form of text or discourse thus coding or discourse analysis methodologies can be employed. Visual images can be analysed by reading their meanings and reflectively disclosing the researcher point of view, perspective, values and background. Interpreting an image is a subjective process, thus a more formal approach to minimise the bias and guarantee objectivity must be used as for the coding process. The stage of theory generation offered by qualitative research methods is linked to Grounded Theory. This is a methodology for developing theories that are grounded in data that is systematically gathered and analysed [14. Theories are derived inductively from the analysis of an underlying study and a reflection and interpretation is conducted on the phenomena under investigation. It can be referred to as a set of procedures for explicating the relationship among pieces of data and a set of descriptive categories from which a plausible explanation of the investigated 
phenomena can be generated. The first step of this methodology is theoretical sampling in which data is collected iteratively until theoretical saturation is reached, that means when the researcher has enough information to describing the underlying phenomena under investigation. Coding is subsequently applied to collected data to disassembling and reassembling it. It first breaks collected data down into manageable chunks, parts such as lines, paragraphs or sections to facilitate meaning and pattern extraction. Examples include open, axial and selective coding techniques. It then employs categorisation to reassemble these parts and produce concepts thus a new understanding that investigates differences and similarities across a number of different cases. Initially, confusion is high, but as the iterative process continues, themes emerge and the analysis becomes more structured and organised. Through the application of constant comparison, core categories are identified, accounting for most of the collected pieces of data and their relationship that far. Eventually, saturation is reached when no new categories are formed, that means when no new insight, categories as well as relationships and properties can be produced. Formally, theoretical saturation is connected to the notion of theoretical completeness that means it is reached when a theory can be shaped and successfully able to explain the data satisfactorily and fully [18].

\section{Extending qualitative data analysis research methods with artificial intelligence}

Qualitative data analysis is key to generate preliminary research hypothesis that can be further tested with quantitative research methods. Nowadays, new and less intrusive data gathering technologies can significantly automatise and speed the acquisition process of qualitative data. Their advantage is that they allow to collect higher dimensional information and larger sample sizes. However, the drawback is that this new multi-dimensional and larger amount of data cannot be inspected qualitatively and individually by qualitative researchers in its entirety. Therefore, there is the need of intelligent solutions to minimise the time and labour demanded to qualitative data analysts to interpret qualitative data, but at the same time to take full advantage of the richness of information and depth of knowledge that can be extracted from it. Artificial intelligence, with its theoretical and practical advances, as well as its novel intelligent applications can offer many solutions to this problem. This section is devoted to the introduction and description of novel methods for qualitative data analysis offered by artificial intelligence and its advances. It is intended to provide qualitative research practitioners with notions and techniques that can be employed to extend their traditional methods for data analysis. To achieve this goal, the reminder of this section reviews intelligent applications, created within the larger field of artificial intelligence, that work on qualitative data. These applications will by organised and grouped by considering the main goals of artificial intelligence (section 2.1. and the type and form of qualitative data being considered (section 2.2). 
Representing qualitative knowledge into a formal form that either humans and computer can interpret is one of the goals of artificial intelligence. 122 and 552 proposed a method for generating semantic networks from text. These types of networks are aimed at representing a knowledge-base that embeds semantic relations between concepts in a graph [125] and thus can help the qualitative researcher to explore knowledge and extract meanings. Similarly, ontologies play an important role in representing and organising knowledge. They are graphs but usually contains fewer formal semantics than semantic networks. They are aimed at representing entities, events, ideas along with their interdependent relations and properties, by using a system of categories [127. For example, 145] introduced a method for deriving domain ontologies from the concept maps semiautomatically generated from textual information for educational purposes. A similar work proposed a method for the automatic acquisition of taxonomies, special concept hierarchies from a text corpus in order to formulate rules and relations in an abstract but concise way and foster knowledge search and reuse [17].

Knowledge representation is strictly connected to reasoning, another important sub-field of artificial intelligence. Knowledge-graphs, ontologies, taxonomies can help organise text into interlinked concepts and categories, providing qualitative researchers with a formal tool to build knowledge and extract rules that can be used in a reasoning system. This is a formal tool that generates conclusions from available knowledge inductively or deductively using an underlying logic [74. Information in reasoning systems can be formalised using the notion of rule, a monological structure that links premises, built upon evidence and/or the knowledge or beliefs of the researcher, to a claim or conclusion. These can be connected to each other also into a dialogical structure, through the notion of conflict and their interaction can reveal the most rationale conclusion/s 67. Reasoning systems can help qualitative researchers to reason over available knowledge characterised by uncertainty, partiality and conflictuality and can reveal special cases in which previous beliefs and intuitions are no longer valid [70. An example of application of formal reasoning can be found in [109, where authors attempted to represent and formally model the ill-defined construct of mental workload [112] or for the prediction of mortality in elderly using fragmented qualitative knowledge related to biomarkers [110111].

Knowledge representation, reasoning systems and their tools can enable qualitative researchers in planning actions or decisions [49. Automated planning supports the realisation of strategies and sequences of action from a complex space of possible solutions. It is strictly related to decision theory and given a description of the possible initial states of the underlying domain under consideration, the possible desired goals and actions, automated planning allows to synthesise a plan that is guaranteed to generate a state which contains the desired goals [90. For example, 53] introduced a system for automatically synthesizing curricula and dynamically constructing learning paths even from disjoint learning objects with the goal of providing learners with a personalised learning solution that 
accounts for their preferences, profile needs and abilities. Similarly, 29] focused on planning learning routes employing a constraint programming approach that required, among other things, to model the profile of learners, to learn concepts and to understand tasks and attain concepts at different competence levels.

Each act, word and gesture of an interviewed participant is important for a qualitative researcher. Machine perception, a subfield of AI, can provide methods for classification and recognition of symbolic human behaviours such as gestures [84 88 and verbal behaviour such as speech 35. The latter not only can be employed to transcribe digital interviews automatically, thus significantly reducing human labour [4612, but it can be used for emotion recognition in speeches 102 138 and dialogs [59, usually tasks for humans. Emotions can be also recognised by automatic analysis of images [26] or hybrid multimodal approaches including images and speeches [10].

A recent article [15] explored the use of machine learning to support qualitative coding through the use of visual tools. In general machine learning can be divided into supervised and unsupervised. The former requires labelled data and it is useful for prediction and forecasting, while the latter is suitable for the exploration of high-dimensional data, its clustering and patterns extraction. These can be related to different grounded theories [41]. According to [87, the former could be related to Glaserian grounded theory [129] because of the availability of ground truth, the labels, equivalent to the Glaser's coding families [33. The latter can be related to the Straussian grounded theory [130] because it is unconstrained to prior theories. Both can be used in different orders and can extend traditional qualitative research methods. For example, on one hand, [1401] used topic modelling for automatic, unsupervised discovery of hidden semantic structures and clusters of similar words, in a text body. Similarly, 36 proposed an unsupervised sentiment extraction approach from textual data. Work on opinion and sentiment analysis is extremely vast $97 / 65 / 13264$. On the other hand, 85] used supervised machine learning to identify the most representative sentences that affected success on Kickstarter, a crowdfunding website where artists and entrepreneurs seek funding, by analysing million phrases and other variables commonly present on these sites. Similarly, 101] and [23] employed supervised machine learning respectively for breast cancer classification via histology images and classification of normal/abnormal brain MRI images. A hybrid approach by 114 firstly used Latent Dirichlet Allocation and Formal Concept Analysis as two unsupervised techniques for respectively extracting topics and deriving a concept hierarchy. The outputs of these approaches were individually used as the input of a machine learning classifier for the classification of short-text documents. Similarly, the whole class of deep learning-based classification techniques can perform automatic feature extraction and learn representations from pictures in a supervised [91] or unsupervised fashion [131. Many other applications exist that segments images [66] or perform sentiment classification from text [133]. 
A method to help researchers to discover patterns from qualitative data has been proposed in [136. A number of semistructured qualitative interviews on the experience of participants in using distance education were conducted. Audio recordings were transformed into textual transcripts and an automatic natural language processing tool identified relevant first and second order categories. A graph was subsequently developed to associate first and second order categories, and subsequently employed for theme discovery. Natural language processing has been shown to be a potential solution for coding problems [37] and a combination of computational and manual techniques can preserve the strengths of traditional content analysis, but at the same time offering systematic rigour and contextual sensitivity 61. Yan et al. proposed using natural language processing jointly with machine learning to train a classification model of initially labelled codes and subsequently used to automatically generate codes. A human-in-the-loop approach subsequently involves human to supervise and to correct predicted codes and use the outcome of this process to create more robust and accurate models 62. This method allows for a faster categorisation of the unit of a qualitative linguistic corpus. 21] compared two methods for developing rules for extracting coded text. The first included a manual approach in which an expert, in natural language processing, developed rules to extract the coded segments. The second employed machine learning to train models capable of predicting codes. Results showed how the former approach worked better with smaller sample sizes. However, the latter approach achieved better accuracy with larger sample sizes.

\section{Enhancing education, teaching and learning with artificial intelligence for qualitative data analysis}

The discipline of education has a long history and has always attracted many scholars, practitioners and scientists all interested in understanding how us, as humans, learn and how to facilitate the acquisition of knowledge and skills. Education is often directed and involves teachers, lecturers, professors and many other educators who orchestrate classrooms, instructional material, delivery methods and teaching activities. These notions are the key components of pedagogy, the theory and practice of education that aims at understanding learning and supports the growth of learners in various educational contexts with an emphasis of the individual interactions and group dynamics. Various learning theories have emerged in the years, each focusing on different aspects on how knowledge is acquired, maintained and processed during learning. These can be summarised in three main categories: behaviourism, constructivism, cognitivism [117/39|44].

Behaviourism, grounded in psychology, is concerned with observable behaviour of learners. It is a reductionist methodological approach to learning assuming that all types of behaviours, regardless of their complexity, can be reduced to a simple association between external stimulus and response in an environment without considering the internal mental states of learners [141]. 
Constructivism, grounded in the earlier work of Piaget [139] and Vygotsky [48, is focused on how learners construct their knowledge from their experiences. It is grounded on the notion of prior knowledge, affected by the environment and social connections of learners. Constructivists assume that learning is an active constructive process with the learner as the core element of information processing who creates own internal subjective representation, due to their own prior knowledge, by acquiring knowledge in objective reality 128. Cognitivism, grounded in cognitive psychology, focuses on cognition, largely neglected within behaviourism [77. It argues that the way humans think actually affect their behaviour thus this cannot be regarded as behaviour in and of itself. The core notion behind cognitivism is that the mind is seen as a closed box which should be opened and investigated. Cognitivists essentially regard learners as complex information processors who assimilate and expand knowledge through cognitive development absorbing stimuli within different environments and processing them to produce knowledge and support the formation of skills [45 195]. The learning theories have been used and compared many times 24] within the field of instructional design 8194. With the technological progress, a new theory has recently emerged: connectivism 34. It essentially focused on the Internet technologies and how these have created a new class of opportunities for learners to share information across themselves through the World Wide Web.

Artificial intelligence, as previously mentioned, with its theoretical and practical advances, as well as its novel intelligent applications can offer many solutions to the problem of qualitative data analysis and extraction of meanings. Unfortunately, their use in educational contexts is often under explored. This is mainly caused by the background of instructions, lecturers and educational practitioners which is very often less technical than that of computer scientists, engineers and other practitioners working within artificial intelligence. The former usually tend to employ traditional qualitative research methods for data gathering and analysis, while the latter usually prefer quantitative research methods. However, in science, making full use of the advantages of these two methods is known to enrich data analysis, enhance meaning and knowledge extraction as well as supporting hypothesis generation, testing and confirmation 2018. This section is devoted to the description of how the methods, developed so far within artificial intelligence that deal with qualitative data, as described in section 3 , can empower education, teaching and learning. In particular, as a third-level instructor, I am interested in exploring the possibilities of how these methods can support my work in the classroom. Thus I focus on describing a set of potential applications of these methods for enhancing teaching and learning in the classroom with a reference to the underlying pedagogical theory, as described in section 4 .

The main goal of behaviourism is to observe the behaviours of learners over time. Arranging the setting of a typical third-level classroom in a way that an instructor can observe the behaviour of the learners against the learning strategy employed is not a trivial task. Learners are more inclined to change their 
behaviours and engage in the classroom if they experience positive feelings during learning as well as from the approval from their fellow learners. They tend not to engage in behaviours that are unpleasant but they are rather inclined to form habits from engaging and pleasant behaviours. AI solutions for face and emotion recognition can be employed by instructors in real-time in the classroom and can serve as significant sources of data for improving instructional design and observing behaviours over time. For example, 27] implemented a neural network for recognising emotions based on facial expressions. Recognising emotions in real-time in the classroom can help an instructor understand the mental states of learners and adjust content delivery accordingly or as a means to temporally break the activities. The solution can be implemented with minimal effort as only a few cameras, placed in different corners of a room, are required for capturing faces and recognising emotions. The bottleneck is represented by training the neural network that can take many days. However, with novel transfer learning solutions, within the sub-field of machine learning, pretrained networks specialised in specific tasks, in this case face recognition and then emotion recognition, are available and can be easily deployed [96, demonstrating the feasibility of this solution. Change in behaviours can also be inferred by the speech of learners engaged in various activities [119].

Constructivism focuses on understanding how learners construct their knowledge. It emphasises the active and autonomous role of learners while understanding and building knowledge. In a typical classroom, a context with multiple learners, constructivism can be achieved, for instance, by performing collaboration, information exchange and inquiring activities among peers. Activity recognition algorithms, employing audio, video [113] and other qualitative sensor-based data 10755, can help categorise the specific activities each learner can perform individually or in group such as 'speaking', 'gestures', 'listening', 'reading', 'writing' and many others while constructing information. An instructor, through the individual and group analysis of these categories can design and converge to more engaging, democratic and student-centered constructivist activities that enhance learning and maximise engagement and experience. Dialogs and discussions among learners represent the most powerful constructivist teaching method, thus promoting it is key 93 . By employing natural language processing techniques from qualitative speech or textual data can help reconstruct the flows of arguments made among learners as part of discussions while constructively building knowledge [56]. Additionally, it can help identify clusters of qualitative data and help instructors build formal representations for an argumentative piece of text [100] that can be used for assessing learning.

The key objective of cognitivism is to understand how the human brain actually learns. It involves the exploration of how we memorise and represent knowledge, how we tackle problem solving and acquire skills, as well as how we develop intelligence. It is connected to the notion of cognitive development and it relies on five principles: remembering, understanding, applying, evaluating, and 
creating. An instructor can assess each of these skills for each learner in the classroom, for example by means of text production. Initially, a learner can be asked to write a paragraph to sketch the content of the previous class (remembering). Subsequently, s/he can focus on writing another paragraph towards interpreting that content through a textual debate, a list of examples and any form of classification of information (understanding). The learner can then focus on problem solving and, for instance, textually answer questions and solve a specific problem (applying). Then, the instructor can ask each student to graphically illustrate certain information, as for instance employing concept maps 92 or producing a pros and cons list (creating). Eventually, the learner can be asked to write a short manual or guidebook to demonstrate important information (creating). Once the textual content for each student is available, the instructor can then employ natural language processing techniques from artificial intelligence to discover similarities against own expert textual information. For instance, semantic similarity techniques can be employed on the text produced by learners and the instructor [118/19/82 and can be used as a form of formative assessment for each of the five aforementioned principles. Similarly, the instructor can employ graph-based methods for assessing conceptual similarity [57/78] of own representations of instructional material and those produced by learners [115]. Another interesting application of cognitivism is the assessment of cognitive load of learners during various educational activities $95 \mid 73$. Assessing cognitive load can be done through the analysis of the speech from learners [144, the evolution of their emotional states as assessed via emotion recognition [5] as well as their pupil dilation and movements [16] via image processing. Many other methods exist for assessing cognitive load but they are mainly quantitative [71|112]68|86]69].

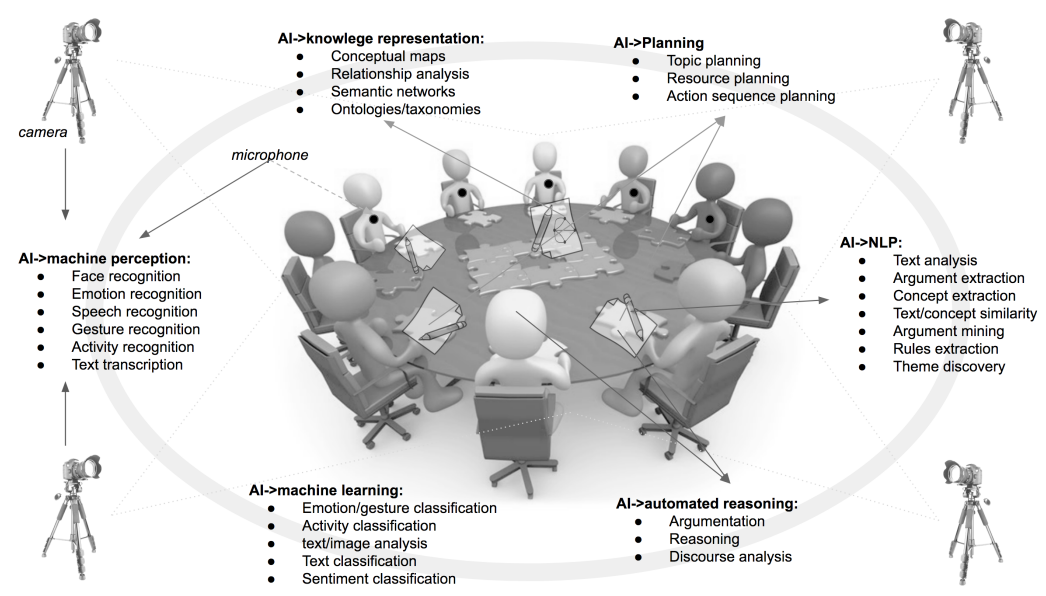

Fig. 2: A summary of the solutions developed within artificial intelligence for empowering qualitative research, its data analysis methods in education, teaching and learning in the classroom 
In summary, qualitative research methods can greatly benefit from the application of theoretical methods, practical tools and solutions developed within the field of artificial intelligence. Catching up with the plethora of theoretical and practical advances of this fast growing field is very difficult even for those researchers working in the field itself, thus it is not expected that a qualitative research practitioner and scholar can fully cope with it. However, given the fact that artificial intelligence is omnipresent and fully part of our daily life, the suggestion is to encourage educational practitioners, instructors and social scientists, mainly employing traditional qualitative research methods, to embrace it and further explore and employ its methods. To achieve this goal, on one hand, scholars in artificial intelligence should work on providing researchers with less technical knowledge with richer explanations and qualitative descriptions of their own methods. On the other hand, researchers, mainly adopting qualitative re-

search methods, should expand their technical knowledge and devote effort on learning those formal concepts and methods offered by artificial intelligence.

\section{References}

1. Arora, S., Ge, R., Halpern, Y., Mimno, D., Moitra, A., Sontag, D., Wu, Y., Zhu, M.: A practical algorithm for topic modeling with provable guarantees. In: International Conference on Machine Learning. pp. 280-288 (2013)

2. Auerbach, C., Silverstein, L.B.: Qualitative data: An introduction to coding and analysis. NYU press (2003)

3. Bahrammirzaee, A.: A comparative survey of artificial intelligence applications in finance: artificial neural networks, expert system and hybrid intelligent systems. Neural Computing and Applications 19(8), 1165-1195 (2010)

4. Bartlett, M.S., Littlewort, G., Frank, M.G., Lainscsek, C., Fasel, I.R., Movellan, J.R.: Recognizing facial expression: Machine learning and application to spontaneous behavior. In: 2005 IEEE Computer Society Conference on Computer Vision and Pattern Recognition (CVPR 2005), 20-26 June 2005, San Diego, CA, USA. pp. 568-573 (2005)

5. Berggren, N., Koster, E.H., Derakshan, N.: The effect of cognitive load in emotional attention and trait anxiety: An eye movement study. Journal of cognitive psychology 24(1), 79-91 (2012)

6. Bishop, C.M.: Pattern recognition and machine learning, 5th Edition. Information science and statistics, Springer (2007)

7. Bogdan, R., Biklen, S.K.: Qualitative research for education. Allyn \& Bacon Boston, MA (1997)

8. Brannen, J.: Mixing methods: Qualitative and quantitative research. Routledge (2017)

9. Buchanan, B.G.: A (very) brief history of artificial intelligence. Ai Magazine 26(4), 53-53 (2005)

10. Busso, C., Deng, Z., Yildirim, S., Bulut, M., Lee, C.M., Kazemzadeh, A., Lee, S., Neumann, U., Narayanan, S.: Analysis of emotion recognition using facial expressions, speech and multimodal information. In: Proceedings of the 6 th international conference on Multimodal interfaces. pp. 205-211. ACM (2004)

11. Caridi, M., Cavalieri, S.: Multi-agent systems in production planning and control: an overview. Production Planning \& Control 15(2), 106-118 (2004) 
12. Chandler, R., Anstey, E., Ross, H.: Listening to voices and visualizing data in qualitative research: Hypermodal dissemination possibilities. Sage Open 5(2), $2158244015592166(2015)$

13. Chang, R., Wang, F., You, P.: A survey on the development of multi-touch technology. In: 2010 Asia-Pacific Conference on Wearable Computing Systems. pp. 363-366. IEEE (2010)

14. Charmaz, K., Belgrave, L.L.: Grounded theory. The Blackwell encyclopedia of sociology (2007)

15. Chen, N.C., Drouhard, M., Kocielnik, R., Suh, J., Aragon, C.R.: Using machine learning to support qualitative coding in social science: Shifting the focus to ambiguity. ACM Transactions on Interactive Intelligent Systems (TiiS) 8(2), 9 (2018)

16. Chen, S., Epps, J.: Using task-induced pupil diameter and blink rate to infer cognitive load. Human-Computer Interaction 29(4), 390-413 (2014)

17. Cimiano, P., Hotho, A., Staab, S.: Learning concept hierarchies from text corpora using formal concept analysis. Journal of artificial intelligence research 24, 305339 (2005)

18. Cohen, L., Manion, L., Morrison, K.: Research methods in education. routledge (2002)

19. Corley, C., Mihalcea, R.: Measuring the semantic similarity of texts. In: Proceedings of the ACL workshop on empirical modeling of semantic equivalence and entailment. pp. 13-18. Association for Computational Linguistics (2005)

20. Creswell, J.W.: Educational research: Planning, conducting, and evaluating quantitative. Prentice Hall Upper Saddle River, NJ (2002)

21. Crowston, K., Allen, E.E., Heckman, R.: Using natural language processing technology for qualitative data analysis. International Journal of Social Research Methodology 15(6), 523-543 (2012)

22. Cruz, J.A., Wishart, D.S.: Applications of machine learning in cancer prediction and prognosis. Cancer Informatics 2, 117693510600200030 (2006)

23. El-Dahshan, E.S.A., Hosny, T., Salem, A.B.M.: Hybrid intelligent techniques for mri brain images classification. Digital Signal Processing 20(2), 433-441 (2010)

24. Ertmer, P.A., Newby, T.J.: Behaviorism, cognitivism, constructivism: Comparing critical features from an instructional design perspective. Performance improvement quarterly 6(4), 50-72 (1993)

25. Fairchild, K.M., Poltrock, S.E., Furnas, G.W.: Semnet: Three-dimensional graphic representations of large knowledge bases. In: Cognitive science and its applications for human-computer interaction, pp. 215-248. Psychology Press (2013)

26. Fasel, B., Luettin, J.: Automatic facial expression analysis: a survey. Pattern recognition 36(1), 259-275 (2003)

27. Filko, D., Martinović, G.: Emotion recognition system by a neural network based facial expression analysis. automatika 54(2), 263-272 (2013)

28. Forsyth, D.A., Ponce, J.: Computer vision: a modern approach. Prentice Hall Professional Technical Reference (2002)

29. Garrido, A., Onaindia, E., Sapena, O.: Planning and scheduling in an e-learning environment. a constraint-programming-based approach. Engineering Applications of Artificial Intelligence 21(5), 733-743 (2008)

30. Gee, J.P.: An introduction to discourse analysis: Theory and method. Routledge (2004)

31. Ghallab, M., Nau, D., Traverso, P.: Automated Planning: theory and practice. Elsevier (2004) 
32. Gil, Y., Greaves, M., Hendler, J., Hirsh, H.: Amplify scientific discovery with artificial intelligence. Science 346(6206), 171-172 (2014)

33. Glaser, B.G.: The grounded theory perspective III: Theoretical coding. Sociology Press (2005)

34. Goldie, J.G.S.: Connectivism: A knowledge learning theory for the digital age? Medical teacher 38(10), 1064-1069 (2016)

35. Graves, A., Mohamed, A.r., Hinton, G.: Speech recognition with deep recurrent neural networks. In: 2013 IEEE international conference on acoustics, speech and signal processing. pp. 6645-6649. IEEE (2013)

36. Greene, S., Resnik, P.: More than words: Syntactic packaging and implicit sentiment. In: Proceedings of human language technologies: The 2009 annual conference of the north american chapter of the association for computational linguistics. pp. 503-511. Association for Computational Linguistics (2009)

37. Grimmer, J., Stewart, B.M.: Text as data: The promise and pitfalls of automatic content analysis methods for political texts. Political analysis 21(3), 267$297(2013)$

38. Hamet, P., Tremblay, J.: Artificial intelligence in medicine. Metabolism 69, S36S40 (2017)

39. Harasim, L.: Learning theory and online technologies. Routledge (2017)

40. Hassabis, D., Kumaran, D., Summerfield, C., Botvinick, M.: Neuroscienceinspired artificial intelligence. Neuron 95(2), 245-258 (2017)

41. Heath, H., Cowley, S.: Developing a grounded theory approach: a comparison of glaser and strauss. International journal of nursing studies 41(2), 141-150 (2004)

42. Hinton, G., Deng, L., Yu, D., Dahl, G., Mohamed, A.r., Jaitly, N., Senior, A., Vanhoucke, V., Nguyen, P., Kingsbury, B., Sainath, T.: Deep neural networks for acoustic modeling in speech recognition. IEEE Signal Processing Magazine 29, 82-97 (November 2012)

43. Huang, W., Nakamori, Y., Wang, S.: Forecasting stock market movement direction with support vector machine. Computers \& OR 32, 2513-2522 (2005)

44. Illeris, K.: Contemporary theories of learning: learning theorists... in their own words. Routledge (2018)

45. Instruction, D.: " paradigm shifts in designed instruction: From behaviorism to cognitivism to constructivism. Educational technology p. 13 (1993)

46. Johnson, B.E.: The speed and accuracy of voice recognition software-assisted transcription versus the listen-and-type method: A research note. Qualitative Research 11(1), 91-97 (2011)

47. Jordan, M.I., Mitchell, T.M.: Machine learning: Trends, perspectives, and prospects. Science 349(6245), 255-260 (2015)

48. Kalina, C., Powell, K.: Cognitive and social constructivism: Developing tools for an effective classroom. Education 130(2), 241-250 (2009)

49. Kane, M., Trochim, W.M.: Concept mapping for planning and evaluation, vol. 50. Sage Publications Thousand Oaks, CA (2007)

50. Kempen, G.: Natural language generation: New results in artificial intelligence, psychology and linguistics, vol. 135. Springer Science \& Business Media (2012)

51. Koehn, P., Hoang, H., Birch, A., Callison-Burch, C., Federico, M., Bertoldi, N., Cowan, B., Shen, W., Moran, C., Zens, R., Dyer, C., Bojar, O., Constantin, A., Herbst, E.: Moses: Open source toolkit for statistical machine translation. In: Proceedings of the 45th Annual Meeting of the ACL on Interactive Poster and Demonstration Sessions. pp. 177-180. ACL '07, Association for Computational Linguistics, Stroudsburg, PA, USA (2007), http://dl.acm.org/citation.cfm?id= 1557769.1557821 
52. Kok, S., Domingos, P.: Extracting semantic networks from text via relational clustering. In: Joint European Conference on Machine Learning and Knowledge Discovery in Databases. pp. 624-639. Springer (2008)

53. Kontopoulos, E., Vrakas, D., Kokkoras, F., Bassiliades, N., Vlahavas, I.: An ontology-based planning system for e-course generation. Expert Systems with Applications 35(1-2), 398-406 (2008)

54. Krippendorff, K.: Content analysis: An introduction to its methodology. Sage publications (2018)

55. Lara, O.D., Labrador, M.A.: A survey on human activity recognition using wearable sensors. IEEE communications surveys \& tutorials 15(3), 1192-1209 (2012)

56. Lawrence, J., Reed, C.: Argument mining using argumentation scheme structures. In: COMMA. pp. 379-390 (2016)

57. Leake, D.B., Maguitman, A.G., Cañas, A.J.: Assessing conceptual similarity to support concept mapping. In: FLAIRS Conference. pp. 168-172 (2002)

58. LeCun, Y., Bengio, Y., Hinton, G.: Deep learning. nature 521(7553), 436 (2015)

59. Lee, C.M., Narayanan, S.S., et al.: Toward detecting emotions in spoken dialogs. IEEE transactions on speech and audio processing 13(2), 293-303 (2005)

60. Legard, R., Keegan, J., Ward, K.: In-depth interviews. Qualitative research practice: A guide for social science students and researchers 6(1), 138-169 (2003)

61. Lewis, S.C., Zamith, R., Hermida, A.: Content analysis in an era of big data: A hybrid approach to computational and manual methods. Journal of broadcasting \& electronic media 57(1), 34-52 (2013)

62. Liew, J.S.Y., McCracken, N., Zhou, S., Crowston, K.: Optimizing features in active machine learning for complex qualitative content analysis. In: Proceedings of the ACL 2014 Workshop on Language Technologies and Computational Social Science. pp. 44-48 (2014)

63. Lincoln, Y.S., Denzin, N.K.: The handbook of qualitative research. Sage (2000)

64. Liu, B.: Sentiment analysis and opinion mining. Synthesis lectures on human language technologies 5(1), 1-167 (2012)

65. Liu, B., et al.: Sentiment analysis and subjectivity. Handbook of natural language processing 2(2010), 627-666 (2010)

66. Liu, F., Lin, G., Shen, C.: Crf learning with cnn features for image segmentation. Pattern Recognition 48(10), 2983-2992 (2015)

67. Longo, L.: Argumentation for knowledge representation, conflict resolution, defeasible inference and its integration with machine learning. In: Machine Learning for Health Informatics, pp. 183-208. Springer (2016)

68. Longo, L.: Subjective usability, mental workload assessments and their impact on objective human performance. In: IFIP Conference on Human-Computer Interaction. pp. 202-223. Springer (2017)

69. Longo, L.: On the reliability, validity and sensitivity of three mental workload assessment techniques for the evaluation of instructional designs: A case study in a third-level course. In: Proceedings of the 10th International Conference on Computer Supported Education, CSEDU 2018, Funchal, Madeira, Portugal, March 15-17, 2018, Volume 2. pp. 166-178 (2018)

70. Longo, L., Dondio, P.: Defeasible reasoning and argument-based systems in medical fields: An informal overview. In: Computer-Based Medical Systems (CBMS), 2014 IEEE 27th International Symposium on. pp. 376-381. IEEE (2014)

71. Longo, L., Dondio, P.: On the relationship between perception of usability and subjective mental workload of web interfaces. In: 2015 IEEE/WIC/ACM International Conference on Web Intelligence and Intelligent Agent Technology (WIIAT). vol. 1, pp. 345-352. IEEE (2015) 
72. Longo, L., Dondio, P., Barrett, S.: Enhancing social search: a computational collective intelligence model of behavioural traits, trust and time. In: Transactions on computational collective intelligence II, pp. 46-69. Springer (2010)

73. Longo, L., Orru, G.: An evaluation of the reliability, validity and sensitivity of three human mental workload measures under different instructional conditions in third-level education. In: McLaren, B.M., Reilly, R., Zvacek, S., Uhomoibhi, J. (eds.) Computer Supported Education. pp. 384-413. Springer International Publishing, Cham (2019)

74. Lowrance, J.D., Garvey, T.D., Strat, T.M.: A framework for evidential-reasoning systems. In: Classic Works of the Dempster-Shafer Theory of Belief Functions, pp. 419-434. Springer (2008)

75. Lyon, R.F.: Machine hearing: An emerging field [exploratory dsp]. Ieee signal processing magazine 27(5), 131-139 (2010)

76. M., U., M., G.: Ontologies: Principles, methods and applications. The knowledge engineering review 11, 93-136 (1996)

77. Mandler, G.: Origins of the cognitive (r) evolution. Journal of the History of the Behavioral Sciences 38(4), 339-353 (2002)

78. Marshall, B., Chen, H., Madhusudan, T.: Matching knowledge elements in concept maps using a similarity flooding algorithm. Decision Support Systems 42(3), 12901306 (2006)

79. Mayring, P.: Qualitative content analysis. A companion to qualitative research 1, 159-176 (2004)

80. McCulloch, W.S., Pitts, W.: A logical calculus of the ideas immanent in nervous activity. The bulletin of mathematical biophysics 5(4), 115-133 (1943)

81. McLeod, G.: Learning theory and instructional design. Learning Matters 2(3), 35-43 (2003)

82. Mihalcea, R., Corley, C., Strapparava, C., et al.: Corpus-based and knowledgebased measures of text semantic similarity. In: AAAI. vol. 6, pp. 775-780 (2006)

83. Miller, T.: Explanation in artificial intelligence: Insights from the social sciences. Artificial Intelligence (2018)

84. Mitra, S., Acharya, T.: Gesture recognition: A survey. IEEE Transactions on Systems, Man, and Cybernetics, Part C (Applications and Reviews) 37(3), 311324 (May 2007)

85. Mitra, T., Gilbert, E.: The language that gets people to give: Phrases that predict success on kickstarter. In: Proceedings of the 17th ACM conference on Computer supported cooperative work \& social computing. pp. 49-61. ACM (2014)

86. Moustafa, K., Longo, L.: Analysing the impact of machine learning to model subjective mental workload: A case study in third-level education. In: International Symposium on Human Mental Workload: Models and Applications. pp. 92-111. Springer (2018)

87. Muller, M., Guha, S., Baumer, E.P., Mimno, D., Shami, N.S.: Machine learning and grounded theory method: convergence, divergence, and combination. In: Proceedings of the 19th International Conference on Supporting Group Work. pp. 3-8. ACM (2016)

88. Murakami, K., Taguchi, H.: Gesture recognition using recurrent neural networks. In: Proceedings of the SIGCHI conference on Human factors in computing systems. pp. 237-242. ACM (1991)

89. Musante, K., DeWalt, B.R.: Participant observation: A guide for fieldworkers. Rowman Altamira (2010)

90. Nau, D.S.: Current trends in automated planning. AI magazine 28(4), 43-43 (2007) 
91. Ng, H.W., Nguyen, V.D., Vonikakis, V., Winkler, S.: Deep learning for emotion recognition on small datasets using transfer learning. In: Proceedings of the 2015 ACM on international conference on multimodal interaction. pp. 443-449. ACM (2015)

92. Novak, J.D.: Learning, creating, and using knowledge: Concept maps as facilitative tools in schools and corporations. Routledge (2010)

93. Orru, G., Gobbo, F., O'Sullivan, D., Longo, L.: An investigation of the impact of a social constructivist teaching approach, based on trigger questions, through measures of mental workload and efficiency. In: Proceedings of the 10th International Conference on Computer Supported Education, CSEDU 2018, Funchal, Madeira, Portugal, March 15-17, 2018, Volume 2. pp. 292-302 (2018)

94. Orru, G., Longo, L.: Direct instruction and its extension with a community of inquiry: A comparison of mental workload, performance and efficiency. In: Proceedings of the 11th International Conference on Computer Supported Education, CSEDU 2019, Heraklion, Crete, Greece, May 2-4, 2019, Volume 1. pp. 436-444 (2019)

95. Orru, G., Longo, L.: The evolution of cognitive load theory and the measurement of its intrinsic, extraneous and germane loads: A review. In: Longo, L., Leva, M.C. (eds.) Human Mental Workload: Models and Applications. pp. 23-48. Springer International Publishing, Cham (2019)

96. Pan, S.J., Yang, Q.: A survey on transfer learning. IEEE Transactions on knowledge and data engineering 22(10), 1345-1359 (2009)

97. Pang, B., Lee, L., et al.: Opinion mining and sentiment analysis. Foundations and Trendsß in Information Retrieval 2(1-2), 1-135 (2008)

98. Parsons, S.: Qualitative methods for reasoning under uncertainty. MIT Press (2001)

99. Patton, M.Q.: Qualitative research. Encyclopedia of statistics in behavioral science (2005)

100. Peldszus, A., Stede, M.: From argument diagrams to argumentation mining in texts: A survey. International Journal of Cognitive Informatics and Natural Intelligence (IJCINI) 7(1), 1-31 (2013)

101. Petushi, S., Garcia, F.U., Haber, M.M., Katsinis, C., Tozeren, A.: Large-scale computations on histology images reveal grade-differentiating parameters for breast cancer. BMC medical imaging 6(1), 14 (2006)

102. Pierre-Yves, O.: The production and recognition of emotions in speech: features and algorithms. International Journal of Human-Computer Studies 59(1-2), 157$183(2003)$

103. Pinedo, M.L.: Planning and scheduling in manufacturing and services. Springer (2005)

104. Puppe, F.: Systematic introduction to expert systems: Knowledge representations and problem-solving methods. Springer Science \& Business Media (2012)

105. Rahwan, I., Simari, G.R.: Argumentation in artificial intelligence, vol. 47. Springer (2009)

106. Ramesh, A., Kambhampati, C., Monson, J.R., Drew, P.: Artificial intelligence in medicine. Annals of The Royal College of Surgeons of England 86(5), 334 (2004)

107. Ravi, N., Dandekar, N., Mysore, P., Littman, M.L.: Activity recognition from accelerometer data. In: Aaai, Vol. 5. vol. 5, pp. 1541-1546 (2005)

108. Reiter, E., Dale, R.: Building Natural Language Generation Systems. Cambridge University Press, New York, NY, USA (2000) 
109. Rizzo, L., Longo, L.: Inferential models of mental workload with defeasible argumentation and non-monotonic fuzzy reasoning: a comparative study. In: Proceedings of the 2nd Workshop on Advances In Argumentation In Artificial Intelligence, co-located with XVII International Conference of the Italian Association for Artificial Intelligence, $\mathrm{AI}^{3} @ \mathrm{AI}{ }^{*} \mathrm{IA}$ 2018, 20-23 November 2018, Trento, Italy. pp. 11-26 (2018)

110. Rizzo, L., Longo, L.: A qualitative investigation of the explainability of defeasible argumentation and non-monotonic fuzzy reasoning. In: Proceedings for the 26th AIAI Irish Conference on Artificial Intelligence and Cognitive Science Trinity College Dublin, Dublin, Ireland, December 6-7th, 2018. pp. 138-149 (2018)

111. Rizzo, L., Majnaric, L., Longo, L.: A comparative study of defeasible argumentation and non-monotonic fuzzy reasoning for elderly survival prediction using biomarkers. In: International Conference of the Italian Association for Artificial Intelligence. pp. 197-209. Springer (2018)

112. Rizzo, L.M., Longo, L.: Representing and inferring mental workload via defeasible reasoning: a comparison with the nasa task load index and the workload profile. In: Proceedings of the 1st Workshop on Advances In Argumentation In Artificial Intelligence AI3@AI*IA. CEURS (2017)

113. Robertson, N., Reid, I.: A general method for human activity recognition in video. Computer Vision and Image Understanding 104(2-3), 232-248 (2006)

114. Rogers, N., Longo, L.: A comparison on the classification of short-text documents using latent dirichlet allocation and formal concept analysis. In: Proceedings of the 25th Irish Conference on Artificial Intelligence and Cognitive Science, Dublin, Ireland, December 7 - 8, 2017. pp. 50-62 (2017)

115. Rye, J.A., Rubba, P.A.: Scoring concept maps: An expert map-based scheme weighted for relationships. School Science and Mathematics 102(1), 33-44 (2002)

116. Saldaña, J.: The coding manual for qualitative researchers. Sage (2015)

117. Schunk, D.H.: Learning theories an educational perspective sixth edition. Pearson (2012)

118. Seco, N., Veale, T., Hayes, J.: An intrinsic information content metric for semantic similarity in wordnet. In: Ecai. vol. 16, p. 1089 (2004)

119. Shaikh Nilofer, R., Gadhe, R.P., Deshmukh, R., Waghmare, V., Shrishrimal, P.: Automatic emotion recognition from speech signals: A review. International Journal of Scientific \& Engineering Research 6(4) (2015)

120. Shen, W.: Distributed manufacturing scheduling using intelligent agents. IEEE intelligent systems 17(1), 88-94 (2002)

121. Shen, W., Wang, L., Hao, Q.: Agent-based distributed manufacturing process planning and scheduling: a state-of-the-art survey. IEEE Transactions on Systems, Man, and Cybernetics, Part C (Applications and Reviews) 36(4), 563-577 (2006)

122. Smith, A.E.: Automatic extraction of semantic networks from text using leximancer. In: Companion Volume of the Proceedings of HLT-NAACL 2003Demonstrations. pp. 23-24 (2003)

123. Sonka, M., Hlavác, V., Boyle, R.: Image processing, analysis and and machine vision (3. ed.). Thomson (2008)

124. Sowa, J.F.: Knowledge representation: logical, philosophical, and computational foundations. PWS (2000)

125. Sowa, J.F.: Principles of semantic networks: Explorations in the representation of knowledge. Morgan Kaufmann (2014)

126. Spradley, J.P.: Participant observation. Waveland Press (2016)

127. Staab, S., Studer, R.: Handbook on ontologies. Springer Science \& Business Media (2010) 
128. Steffe, L.P., Gale, J.E.: Constructivism in education. Lawrence Erlbaum Hillsdale, NJ (1995)

129. Stern, P.N., Kerry, J., et al.: Glaserian grounded theory. In: Developing Grounded Theory, pp. 55-85. Routledge (2016)

130. Strauss, A., Corbin, J.M.: Grounded theory in practice. Sage (1997)

131. Supratak, A., Li, L., Guo, Y.: Feature extraction with stacked autoencoders for epileptic seizure detection. In: 2014 36th Annual International Conference of the IEEE Engineering in Medicine and Biology Society. pp. 4184-4187. IEEE (2014)

132. Taboada, M., Brooke, J., Tofiloski, M., Voll, K., Stede, M.: Lexicon-based methods for sentiment analysis. Computational linguistics 37(2), 267-307 (2011)

133. Tang, D., Qin, B., Liu, T.: Document modeling with gated recurrent neural network for sentiment classification. In: Proceedings of the 2015 conference on empirical methods in natural language processing. pp. 1422-1432 (2015)

134. Tanimoto, S.: Structured computer vision: machine perception through hierarchical computation structures. Elsevier (2014)

135. Taylor, S.J., Bogdan, R., DeVault, M.: Introduction to qualitative research methods: A guidebook and resource. John Wiley \& Sons (2015)

136. Tierney, P.: A qualitative analysis framework using natural language processing and graph theory. The International Review of Research in Open and Distributed Learning 13(5), 173-189 (2012)

137. Vaughn, S., Schumm, J.S., Sinagub, J.M.: Focus group interviews in education and psychology. Sage (1996)

138. Ververidis, D., Kotropoulos, C.: Emotional speech recognition: Resources, features, and methods. Speech communication 48(9), 1162-1181 (2006)

139. Wadsworth, B.J.: Piaget's theory of cognitive and affective development: Foundations of constructivism. Longman Publishing (1996)

140. Wallach, H.M.: Topic modeling: beyond bag-of-words. In: Proceedings of the 23rd international conference on Machine learning. pp. 977-984. ACM (2006)

141. Watson, J.B.: Behaviorism. Routledge (2017)

142. Williams, A., Katz, L.: The use of focus group methodology in education: Some theoretical and practical considerations, 5 (3). IEJLL: International Electronic Journal for Leadership in Learning 5 (2001)

143. Witz, K.G., Goodwin, D.R., Hart, R.S., Thomas, H.S.: An essentialist methodology in education-related research using in-depth interviews. Journal of Curriculum Studies 33(2), 195-227 (2001)

144. Yin, B., Chen, F., Ruiz, N., Ambikairajah, E.: Speech-based cognitive load monitoring system. In: 2008 IEEE International Conference on Acoustics, Speech and Signal Processing. pp. 2041-2044. IEEE (2008)

145. Zouaq, A., Nkambou, R.: Building domain ontologies from text for educational purposes. IEEE Transactions on learning technologies 1(1), 49-62 (2008) 